\title{
RISE Microscopy: Correlative Raman and SEM Imaging
}

\author{
Ute Schmidt $^{1}$, Olaf Hollricher ${ }^{1}$, Wei Liu ${ }^{2}$, Edward L Principe ${ }^{3}$ \\ 1. WITec GmbH, Ulm, Germany \\ 2. WITec Instruments, Knoxville, TN, USA \\ 3. Tescan USA, Warrendale, PA, USA
}

RISE Microscopy is a novel correlative microscopy technique which combines confocal Raman Imaging

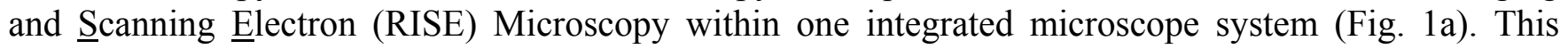
unique combination provides advantages for the microscope user with regard to comprehensive sample characterization: electron microscopy is an excellent technique for visualizing the sample surface structures in the nanometer range; confocal Raman imaging is an established spectroscopic imaging method used for the detection of the chemical and molecular components of a sample with diffraction limited resolution. In contrast to existing combinations, where single Raman spectra are typically collected from few micrometer size areas, the RISE combination allows for the first time diffraction limited confocal Raman imaging on the same sample position as the SEM image was taken. It can also generate 3D-images and depth profiles to visualize the distribution of the molecular compounds within a sample volume. Both analytical methods are fully integrated into the RISE Microscope. Between the different measurements a precise translation stage automatically transfers the sample inside the microscope's vacuum chamber and re-positions it. The integrated RISE software carries out the required parameter adjustments and instrument alignments. The acquired results can then be correlated and the Raman and SEM images overlaid.

The instrument as well as various examples for using this new possibility for correlative confocal Raman imaging with SEM will be presented. A first example of correlative Raman and SEM imaging is presented in Figure $1 b$-d showing the distribution of $\mathrm{TiO}_{2}$ nanoparticles. Fig. 1b shows the SEM image of $\mathrm{TiO}_{2}$ nanoparticles, revealing the presence of two different sizes of the particles. From the same sample area an array of 150x150 complete Raman spectra was acquired with an integration time per spectrum of $37 \mathrm{~ms}$. From this array two distinct spectra were evaluated as shown in Fig. 1c. These two spectra correspond to the two polymorphic phases of $\mathrm{TiO}_{2}$ : the rutile and anatase phases. The correlative Raman-SEM image (Fig. 1d) reveals that the large particles correspond to the rutile phase of $\mathrm{TiO}_{2}$, whereas the small particles consist of anatase $\mathrm{TiO}_{2}$, enabling to differentiate the fine crystallographic structure of the nanoparticles. 


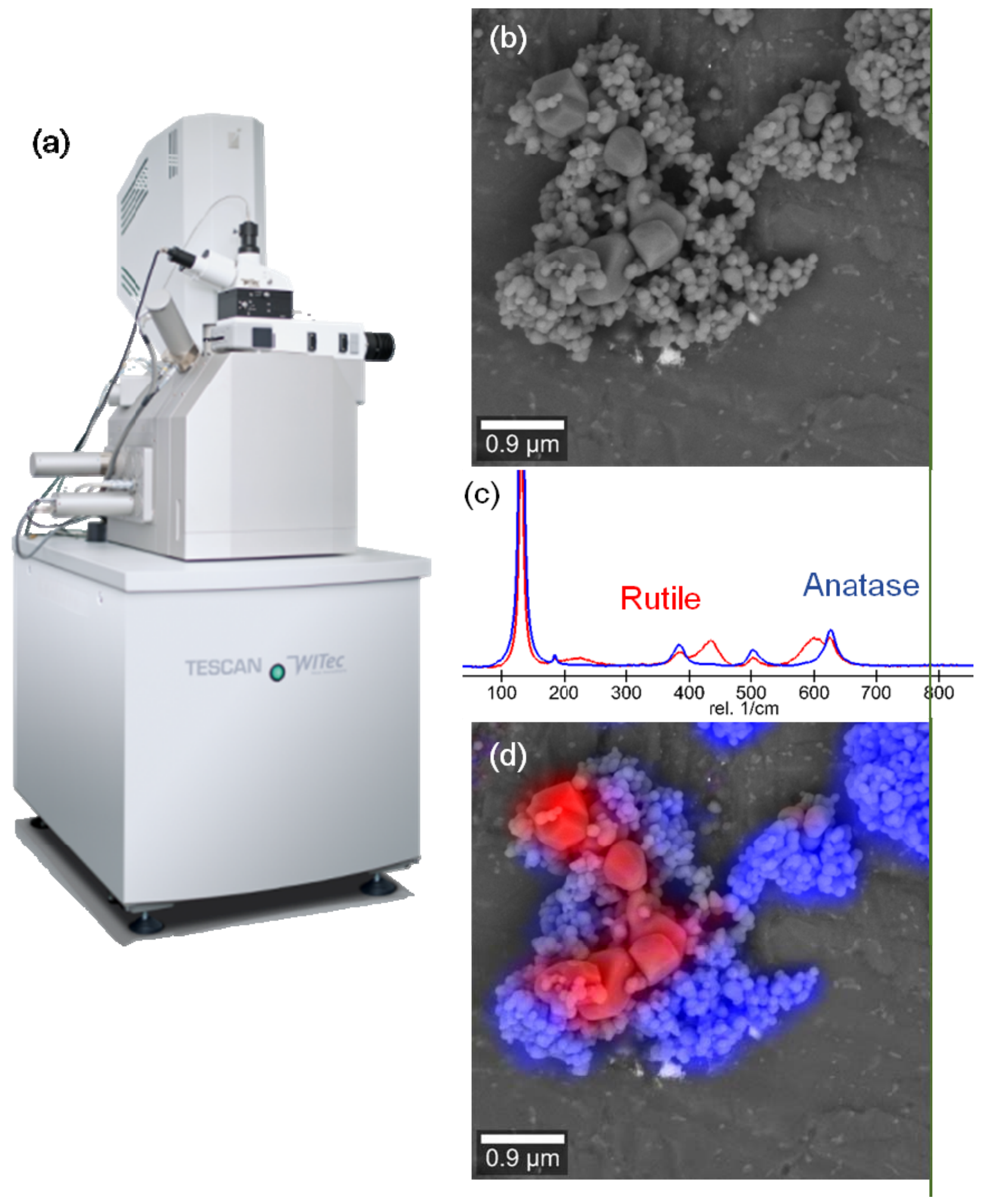

Figure 1. RISE microscope (a), SEM image of $\mathrm{TiO}_{2}$ nanoparticles (b), Raman spectra of rutile and anatase $\mathrm{TiO}_{2}$ (c), and correlative Raman-SEM image (d). 